\title{
Revisiting the method to obtain the mechanical properties of hydrided fuel cladding in the hoop direction
}

\author{
M.A. Martín-Rengel ${ }^{\mathrm{a}, *}$, F.J. Gómez Sánchez ${ }^{\mathrm{b}, 1}$, J. Ruiz-Hervías ${ }^{\mathrm{a}}$, L. Caballero ${ }^{\mathrm{a}}$, A. Valiente ${ }^{\mathrm{a}}$
}

\begin{abstract}
A B S T R A C T
The method reported in the literature to calculate the stress-strain curve of nuclear fuel cladding from ring tensile test is revisited in this paper and a new alternative is presented. In the former method, two universal curves are introduced under the assumption of small strain. In this paper it is shown that these curves are not universal, but material-dependent if geometric nonlinearity is taken into account. The new method is valid beyond small strains, takes geometric nonlinearity into consideration and does not need universal curves. The stress-strain curves in the hoop direction are determined by combining numerical calculations with experimental results in a convergent loop. To this end, ring tensile tests were performed in unirradiated hydrogen-charged samples. The agreement among the simulations and the experimental results is excellent for the range of concentrations tested (up to $2000 \mathrm{wppm}$ hydrogen). The calculated stress-strain curves show that the mechanical properties do not depend strongly on the hydrogen concentration, and that no noticeable strain hardening occurs. However, ductility decreases with the hydrogen concentration, especially beyond 500 wppm hydrogen. The fractographic results indicate that as-received samples fail in a ductile fashion, whereas quasicleavage is observed in the hydrogen-charged samples.
\end{abstract}

\section{Introduction}

Nuclear plants are powered by uranium oxide pellets stacked within long metal tubes which are known as fuel cladding. Their mission is to contain the by-products of the fission process, that is to say radioactive gases and solids. The cladding is the first barrier for the radioactive material. Consequently, it must remain intact during the fuel cycle to ensure a safe operation even under extreme events.

Zirconium alloys are employed as cladding material for nuclear rods. The combination of low neutron absorption, corrosion resistance and good mechanical properties in reactor conditions makes them the best candidates for this application. In spite of these properties, zirconium alloy cladding may became susceptible to failure on the long term. In order to prevent this, nuclear fuel rods are often withdrawn from service even though the fuel itself may have a substantial amount of remaining energy. This is a key issue because if the fuel could be burnt longer in the reactor, while keeping the safety margins, the amount of radioactive waste that must be stored would also be reduced.
Hydrogen embrittlement is one of the key factors in cladding failure. As a consequence of zirconium corrosion in the primary cooling circuit, hydrogen is absorbed inside the cladding and a zirconium oxide layer is formed on its external surface. When the solubility limit is reach, hydrogen will precipitate as zirconium hydrides, with the process being driven by temperature gradients along the cladding wall. It is well known that hydrides may reduce the mechanical properties of the cladding [1-4]. As the structural integrity of the cladding has to be maintained during service and storage operations, it is very important to monitor its mechanical properties at the different steps of the fuel cycle.

The mechanical loads experienced by the cladding (i.e. fuel swelling and fission gases) generate the highest stresses in the hoop direction. The geometry of these thin-walled tubes makes it difficult to obtain the mechanical properties in the hoop direction. In fact, neither of the tests proposed in the literature is able to produce a truly uniaxial stress state in the hoop direction [5]. Consequently, finite element simulations are needed to calculate the stress-strain curve from the experimental results. The ring tensile test has been proposed to determine the mechanical properties of the cladding along the hoop direction. The initial test consisted of applying a force inside a cladding sample, by means of two half cylinders. Later Arsene and Bai [6,7] modified the experimental device to avoid sample bending at the beginning of the test. The same authors proposed a numerical method to obtain the stress-strain 
curve in the hoop direction from the experimental load vs. displacement results and a given friction coefficient between the loading pieces and the sample [1]. This method has been used by different authors [8] with slight modifications. It is based on the existence of two universal curves under small strain hypothesis: the first one correlating the hoop strain with the displacement of the loading piece and the second one correlating the hoop stress with the applied load.

In this work, unirradiated ZIRLO ${ }^{\mathrm{TM}}$ cladding samples with different hydrogen contents (0,150, 250, 500, 1200 and 2000 wppm) were tested by using the ring tensile test. A fractographic analysis was performed on the broken samples to elucidate the fracture micromechanisms. In addition, a novel method is proposed to determine the mechanical properties of cladding in the hoop direction from the ring tensile test results. As non-linear geometry is considered and an iterative procedure is used, universal curves are not needed. The stress-strain curve in the hoop direction is determined by combining numerical calculations with experimental results in a convergent loop. The agreement between calculations and experiments is excellent.

\section{Experimental procedure}

\subsection{Samples and experimental set-up}

The material for this work is unirradiated PWR cladding from ZIRLO $^{\mathrm{TM}}$ alloy [9] with $9.5 \mathrm{~mm}$ outer diameter and 0.57 wall thickness.

The samples for ring tensile tests are machined from the cladding in the shape of rings with a variable height (with two symmetrical gauge sections). As can be seen in Fig. 1, reduced section height is $2 \mathrm{~mm}$.

The experimental device consists of two semi-cylindrical beams which are in contact with the inner surface of the samples. They move relatively to each other when the load is applied. In addition, a centering piece is located inside the ring specimen in order to prevent the notched region from bending at the beginning of the test $[6,7]$. The experimental system is shown in Fig. 2 . The load applied to the beams is measured with a load cell ( $5 \mathrm{kN}$ full scale) and the overall cross-head displacement of the testing machine is recorded. The displacement cross-head speed was $0.3 \mathrm{~mm} / \mathrm{min}$ and the temperature was maintained at $20^{\circ} \mathrm{C}$ for all tests.

The relationship between the hoop stress, in the reduced sections and the applied load, $F_{C}$, is given by the following equation:

$\sigma_{\theta \theta}=\frac{F_{C}}{2 S_{0}}\left(1+f_{C}\right)$

where $S_{0}$ is the area of the reduced section and $f_{C}$ is a factor which depends on the friction coefficient and the radial gap between the
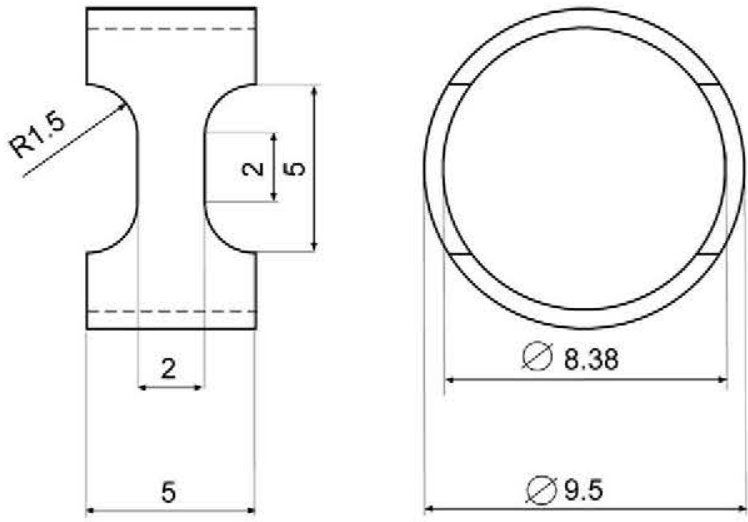

Fig. 1. Dimensions of the ring tensile test samples.

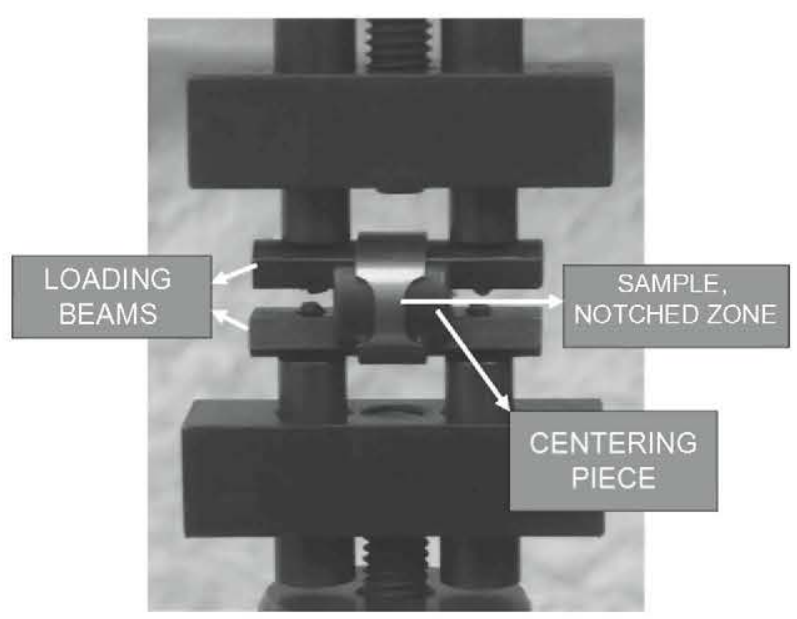

Fig. 2. Experimental loading system employed in the ring tensile test.

sample and the centering piece (see Fig. 2). In the experiments, the radial gap was calculated from precise measurements of the internal diameter of the samples and the centering piece diameter. A profile projector with $1 \mu \mathrm{m}$ resolution was used for this purpose.

The displacement measured by the testing machine comes from two different sources: the sample deformation and the deformability of the loading system. In order to subtract the second contribution, a test was carried out with a thick-walled steel ring $(3.5 \mathrm{~mm}$ wall thickness as compared to $0.57 \mathrm{~mm}$ of the cladding samples). It has been shown in the literature [8] that the displacement, $D$, associated to the loading system in the ring tensile test can be described by the following equation

$D=A \times\left(F_{C}-B\right)^{n}$

where $A, B$ and $n$ depend on the particular loading system employed. In order to obtain these parameters, some tests were carried out with the above mentioned steel ring using the loading system employed in the ring tensile tests. The deformation of the steel ring for the maximum load of a typical ring tensile test (around $1.5 \mathrm{kN}$ ) is negligible.

The deformation at fracture was calculated from the measurement of the initial and final (i.e. after testing) cross-sections at the notched region. To this end, a profile projector with $1 \mu \mathrm{m}$ resolution was used. The deformation at fracture is given by:

$\varepsilon_{r}=\ln \frac{S_{0}}{S_{r}}$

where $S_{0}$ and $S_{r}$ are respectively, the initial and final cross-sections of the notched region. These values can also be used to calculate the area reduction:

$Z=1-\frac{S_{r}}{S_{0}}$

\subsection{Hydrogen charging}

An experimental technique was devised to introduce controlled amounts of hydrogen in the ring samples (up to $2000 \mathrm{wppm}$ hydrogen). Cathodic charging was performed at room temperature in a $\mathrm{KOH}$ aqueous solution. The ring sample was the cathode and a platinum wire coiled around the sample was the anode. During the cathodic charging process, the current density was kept constant and charging time was changed depending on the amount of hydrogen to be introduced in the sample [4]. After charging, samples were heat treated in an argon atmosphere. Samples were maintained at $723 \mathrm{~K}$ for $7 \mathrm{~h}$ and then slowly cooled $(1.2 \mathrm{~K} / \mathrm{min})$. 
This resulted in zirconium hydrides being homogeneously distributed through the cross-section. Hydrogen concentration was measured using the inert gas fusion thermal conductivity detection method with a HORIBA JOBIN-YVON hydrogen analyzer EMGA$621 \mathrm{~W}$.

Hydride morphology and distribution was studied by metallography. Samples were cold-mounted with epoxy resin and then polished up to a $1 \mu \mathrm{m}$ alumina suspension. The last finishing step involved polishing with a mixture of colloidal silica $(0.05 \mu \mathrm{m})$, $\mathrm{H}_{2} \mathrm{O}_{2}$ (30\% vol.) and $\mathrm{HF}(40 \mathrm{wt} . \%$ ) in the proportion (50 v; $10 \mathrm{v} ; 1$ v). After polishing, samples were etched for $90 \mathrm{~s}$ with a solution of $\mathrm{HNO}_{3}$ (69 wt.\%), lactic acid (95 wt.\%) and $\mathrm{HF}(40 \mathrm{wt} . \%$ ) in the proportion ( $48.5 \mathrm{wt}$; $48.5 \mathrm{wt}$; $3 \mathrm{wt}$ ). In such a way the hydride structure was revealed.

The hydrogen charging method produced a homogeneous hydride population oriented along the hoop direction of the samples, as can be seen in Fig. 3. The hydrides are the dark lines observed on the bright zirconium surface. Samples with controlled hydrogen concentrations $(150,250,500,1200$ y $2000 \mathrm{wppm})$ were prepared for the ring tensile tests.

\subsection{Finite element model}

A 3D finite element model was developed with ABAQUS v 6.7-5 commercial code to simulate the ring tensile test. A structured 3D mesh was built by considering one eighth of the sample due to geometrical and loading symmetries. Bilinear parallelepipeds with 20 nodes were employed in the mesh. The element size in the notch zone was $25 \times 25 \times 80 \mu \mathrm{m}$. The mesh employed is shown in Fig. 4.

The loading and boundary conditions were simulated as a solid contact problem. Rigid surfaces were chosen to apply the load (to simulate the loading beams) and to limit the movement of the inner surfaces in the notched zone (simulation of the centering piece constriction). It is a double contact problem with Coulomb-type friction. The friction coefficient employed was 0.125 . The ring tensile test is quite sensitive to this parameter, as shown in the literature [1].

Geometric nonlinearity was considered in the calculations. Geometric nonlinearity refers to the nonlinear stiffness variations caused by large deformations or rotations. Material behaviour was consider elastic-plastic and isotropic. The von Mises yield criterion was used [1].

The method employed to obtain the stress-strain curve from the experimental load vs. displacement results is divided in three steps:

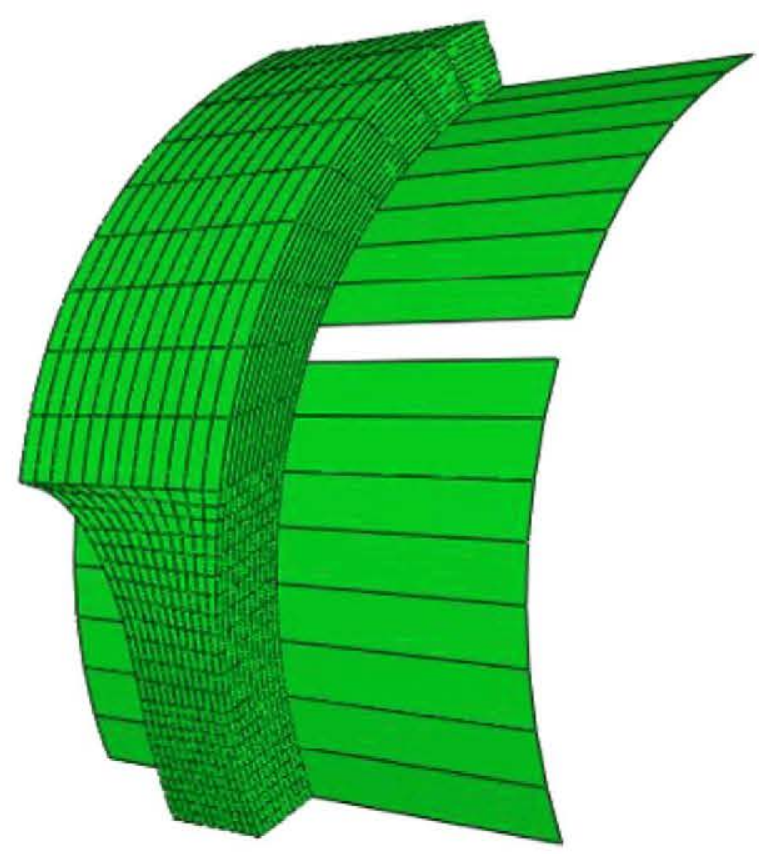

Fig. 4. Structured mesh employed in the model.

- Step 1: A linear-elastic calculation is performed to obtain the Young's modulus. The result is shown in Fig. 5 for the case of a sample with $2000 \mathrm{wppm}$ of hydrogen.

- Step 2: A calculation is performed by choosing a certain value for the elastic limit and assuming that there is no strain hardening. Consequently, the material is considered elastic perfectly plastic in this step. From the simulation results, the curves $k_{t}$ vs $d$ y $\overline{\varepsilon^{p}}$ vs $d$ are obtained. In these curves, $d$ is the displacement of the loading beams, $k_{t}$ is the quotient between the von Mises stress and the nominal stress at the notched zone (i.e. the applied load divided by the cross-section) and $\overline{\varepsilon^{p}}$ the equivalent plastic deformation. From these curves, the relationship between the von Mises stress, $\sigma_{M}$, and the equivalent plastic deformation can be obtained for each value of the displacement.

- Step 3: The curve $\sigma_{M^{-}} \varepsilon^{P}$ obtained in the previous step is fed into the model, and a new calculation carried out to obtain the simulated load vs. displacement curve. This is the first iteration of the process. The process is finished when a good agreement with the experimental results is achieved.

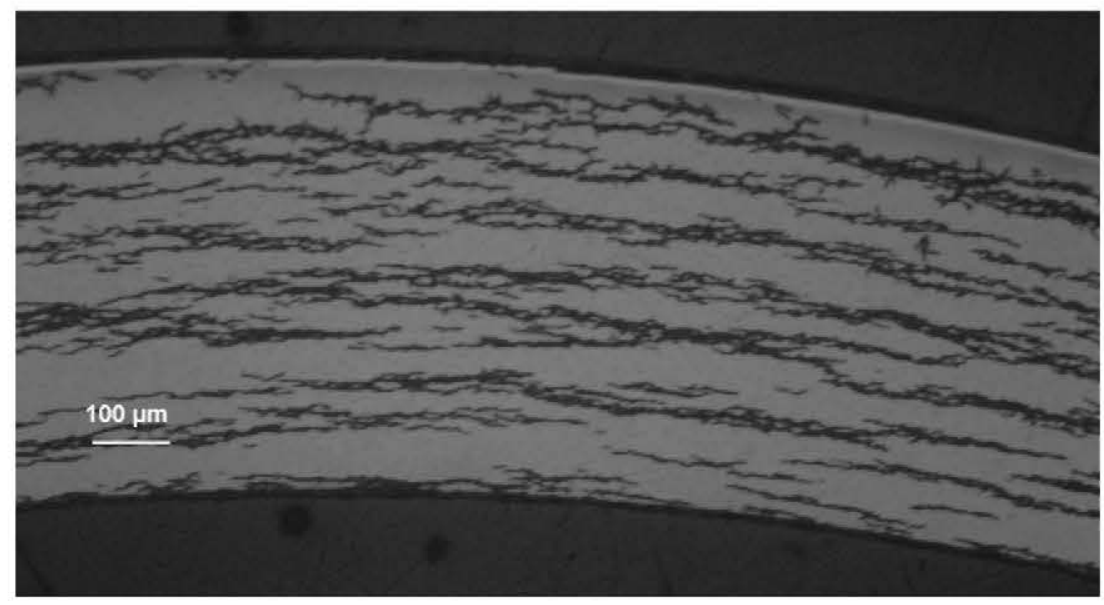

Fig. 3. Hydride distribution in a sample with $500 \mathrm{wppm}$ of hydrogen. 


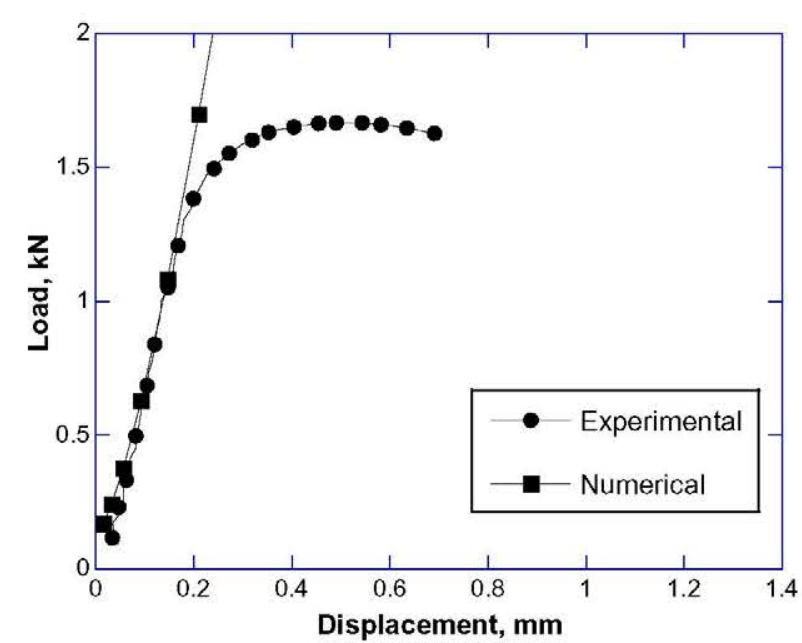

Fig. 5. Linear-elastic calculation to obtain Young's modulus (sample with 2000 wppm of hydrogen).

A remarkable agreement is found with this method in only five iterations. As an example, the experimental and numerical results are compared at the first iteration in Fig. 6 and after five iterations in Fig. 7.

\subsection{Fractography of broken samples}

The fracture surfaces of broken samples (after ring tensile test) were observed by scanning electron microscopy (SEM) by using a JEOL JSM-6300 microscope. The main objective was to investigate the effect of the hydrogen content on the fracture micromechanisms. Thus, samples without hydrogen (as received) and charged with $2000 \mathrm{wppm}$ were studied.

In the as-received samples, the macroscopic fracture (located in the notched region) takes place at the approximately $45^{\circ}$ from the hoop direction of the cladding, as shown in Fig. 8. The fracture surface is located close to the centre of the notched region and necking can be observed close to it. The fracture surface corresponding to the zone enclosed by a circle at the left hand side of Fig. 8 is shown in Fig. 9 at higher magnification. The fracture micromechanism is dominated by nucleation, growth and coalescence of microvoids, which is characteristic of ductile fracture.

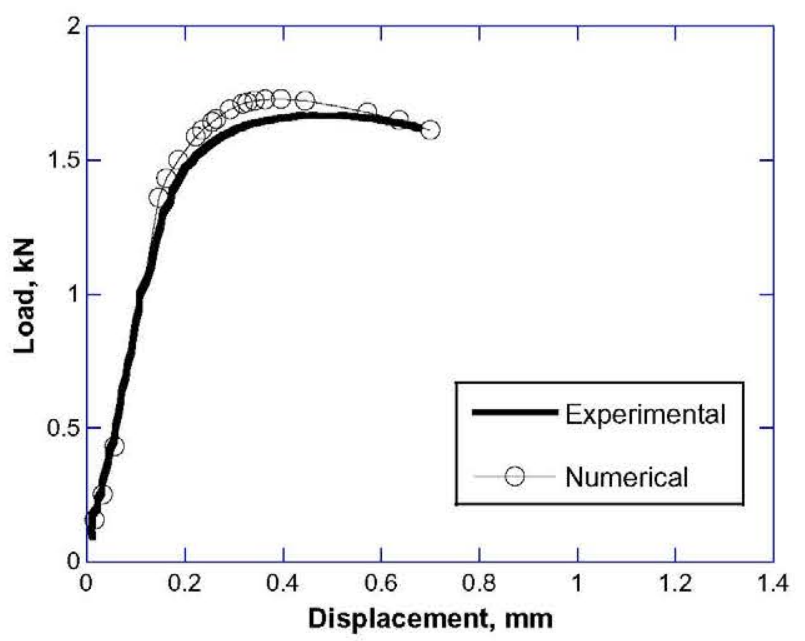

Fig. 6. Experimental and numerical load vs. displacement results, first iteration (sample with 2000 wppm of hydrogen).

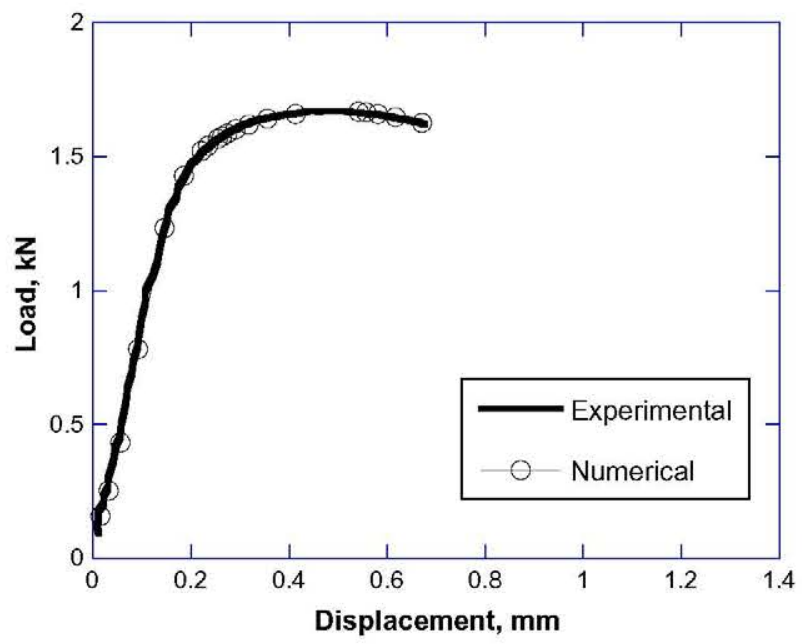

Fig. 7. Experimental and numerical load vs. displacement results, fifth iteration (sample with 2000 wppm of hydrogen).

The macroscopic fracture of samples charged with 2000 wppm of hydrogen is depicted in Fig. 10. In this case, fracture is perpendicular to the hoop direction and takes place close to the filled radius and far from the centre of the notched region. In addition, parallel microcracks are observed at the surface, which correspond to the failure of the oxide layer produced at this hydrogen level. The portion of the fracture surface surrounded by a circle (at the right hand side of Fig. 10) is shown in Fig. 11 at higher magnification. The structure is different from that observed in the asreceived samples (compare Figs. 9 and 11). In this case, the fracture surface consists of bands with faceted surfaces. They have local orientations that suggest a possible relationship with crystallographic planes, which is consistent with a quasi-cleavage micromechanism. The bands are separated by regions where ductile shearing is observed. In addition, other regions show nucleation, growth and coalescence of microvoids (at the left hand side of Fig. 11) In this case, fracture occurs without prior necking of the notched region, which is consistent with a brittle failure mode. This is clearly noticeable when Figs. 8 and 10 are compared.

\section{Results and discussion}

As has been explained above, Arsene and Bai [6,7] proposed a numerical method to obtain the stress-strain curve based on two universal curves. In order to check this hypothesis, several calculations were performed with different materials, namely $E, F, Y$ and $W$, whose mechanical properties are given in Table 1 . The value of the $f_{C}$ parameter (see Eq. (1)) as function of displacement is depicted in Fig. 12 for the above mentioned materials.

In Table $1, E$ is the Young's module, $v$ is the Poisson's coefficient, $\sigma_{0.2}$ is the elastic limit at $0.2 \%$ of deformation, and $h$ is a parameter related to strain hardening ( $h=0$ means no strain hardening).

It can be seen that if non-linear geometry is considered, the curves are fairly different with respect to the ones without non-linear geometry. In fact, only for displacement values smaller than $0.1 \mathrm{~mm}$ the curve can be considered universal, i.e. materialindependent.

The experimental and numerically simulated load vs. displacement results for the ring tensile test are shown in Figs. 13-18. The hydrogen concentration in the samples ranges from 0 wppm (asreceived) to $2000 \mathrm{wppm}$. In all cases, five iterations with the model proposed in this paper were performed.

The agreement of the simulations with the experimental data is excellent, as can be seen in Figs. 13-18. It seems that the calculated 


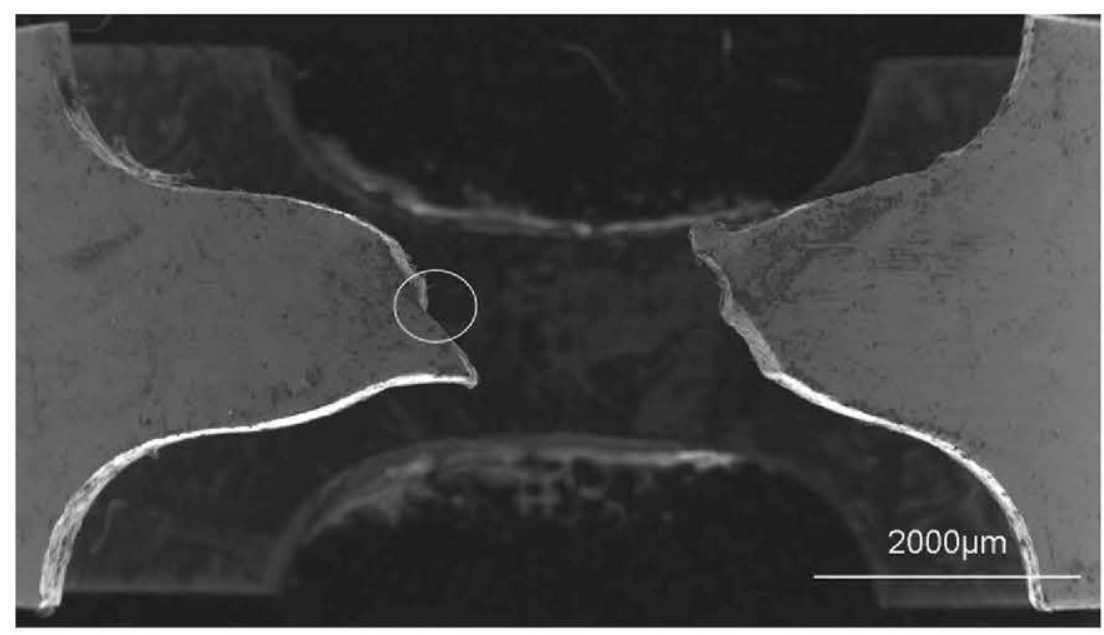

Fig. 8. Macrofractography of an as-received sample (SEM image).

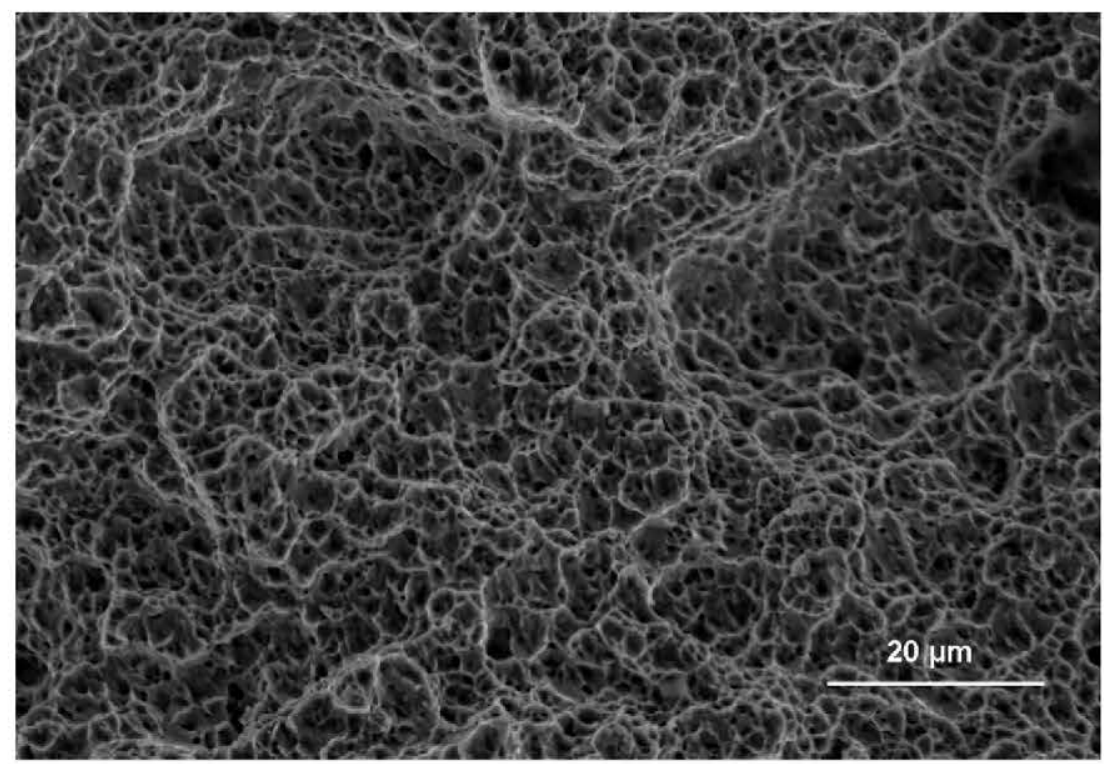

Fig. 9. Detail of the fracture surface for an as-received sample (SEM image).

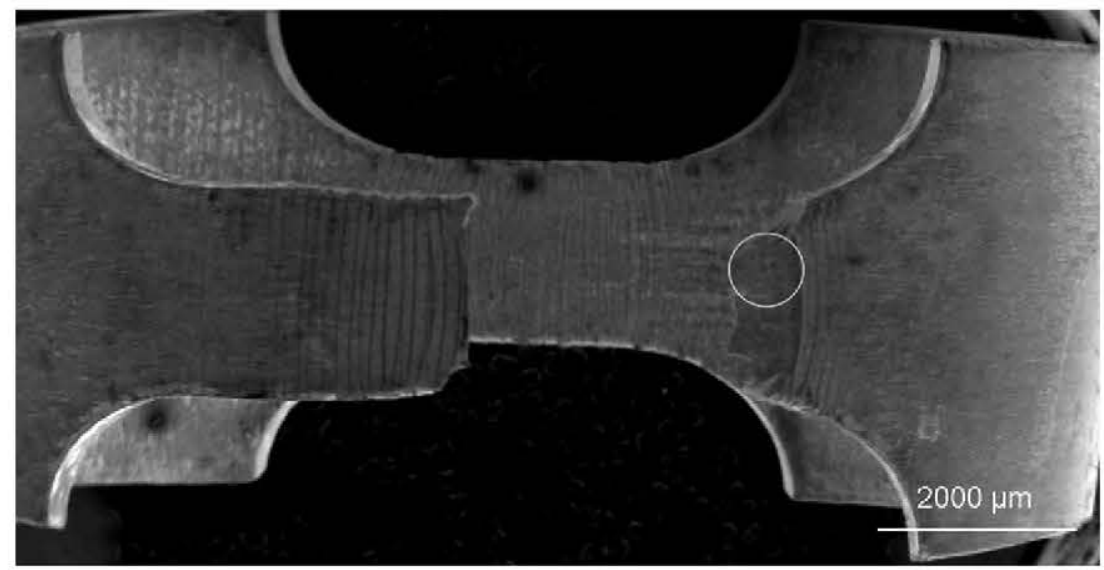

Fig. 10. Macrofractography of sample with $2000 \mathrm{wppm}$ of hydrogen (SEM image). 


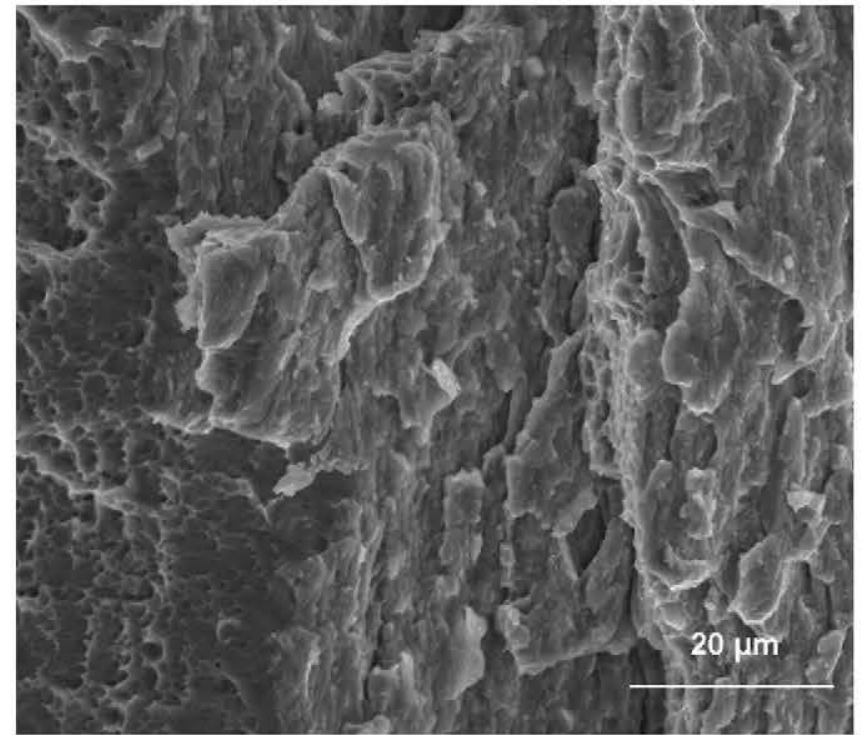

Fig. 11. Detail of the fracture surface for a sample with $2000 \mathrm{wppm}$ of hydrogen (SEM image).

Table 1

Mechanical properties of the materials employed in Fig. 9.

\begin{tabular}{lllcl}
\hline & $E(\mathrm{GPa})$ & $v$ & $\sigma_{0.2}(\mathrm{MPa})$ & $h(\mathrm{MPa}) h=\frac{\sigma(4 \%)-\sigma(10 \%)}{0.06}$ \\
\hline$E$ & 60 & 0.37 & 700 & 275 \\
$F$ & 60 & 0.37 & 500 & 200 \\
$W$ & 60 & 0.37 & 1000 & 0 \\
$Y$ & 60 & 0.37 & 500 & 0 \\
\hline
\end{tabular}

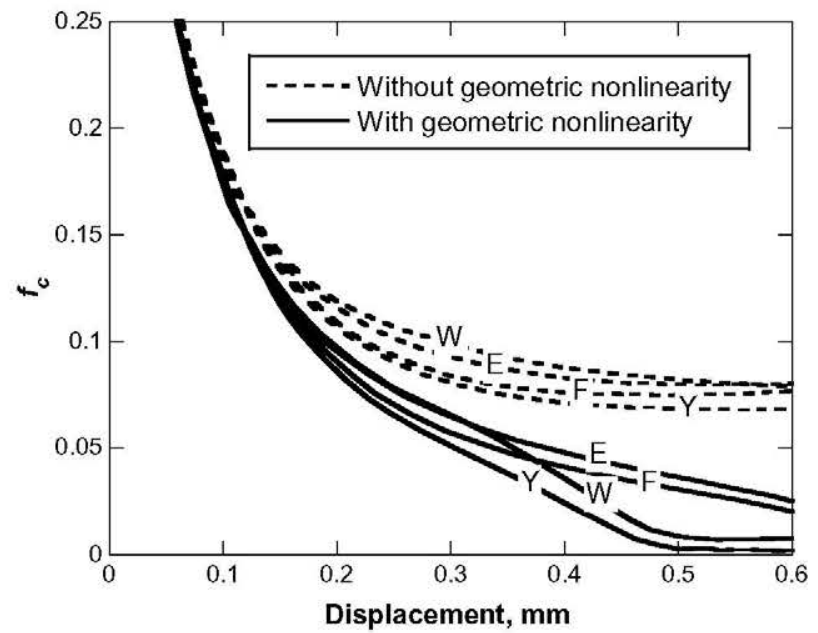

Fig. 12. Calculated $f_{C}$ vs. displacement curves for different materials (see Table 1).

Young's modulus does not change with the hydrogen concentration up to 2000 wppm, with its value being equal to $88 \mathrm{GPa}$. As the hydrogen concentration is increased, the total displacement at fracture decreases. This is an indication of the lower ductility of the material associated with the presence of hydrides. However, the maximum load does not change significantly with the hydrogen concentration. Only for 2000 wppm a slight decrease is found.

The calculated von Mises stress vs. equivalent plastic strain is depicted in Fig. 19 for the hydrogen concentrations investigated. The curves corresponding to the different concentrations are very

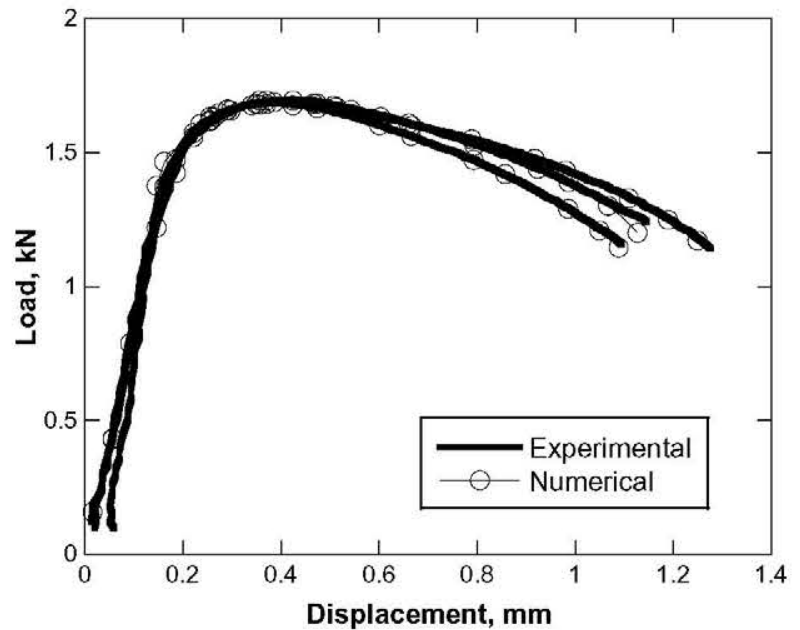

Fig. 13. Experimental and numerical load vs. displacement curve, 0 wppm.

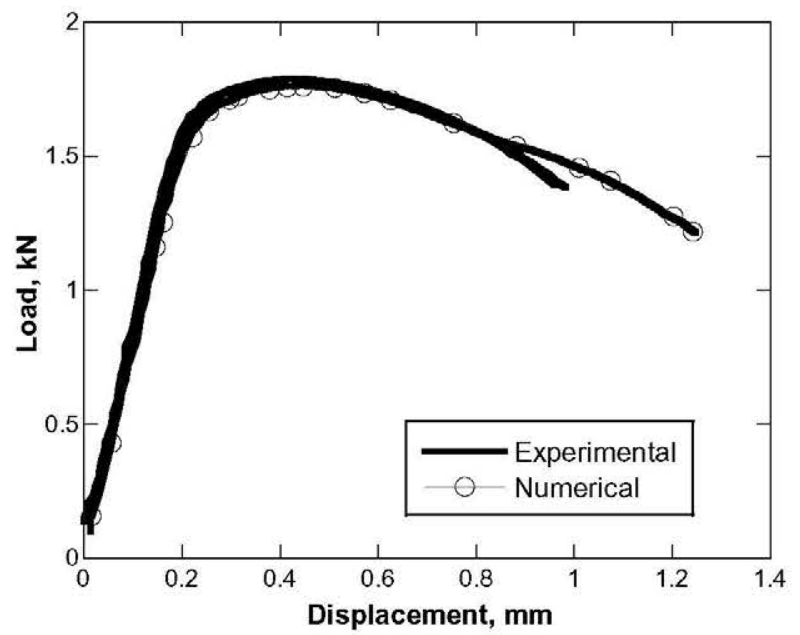

Fig. 14. Experimental and numerical load vs. displacement curve, 150 wppm.

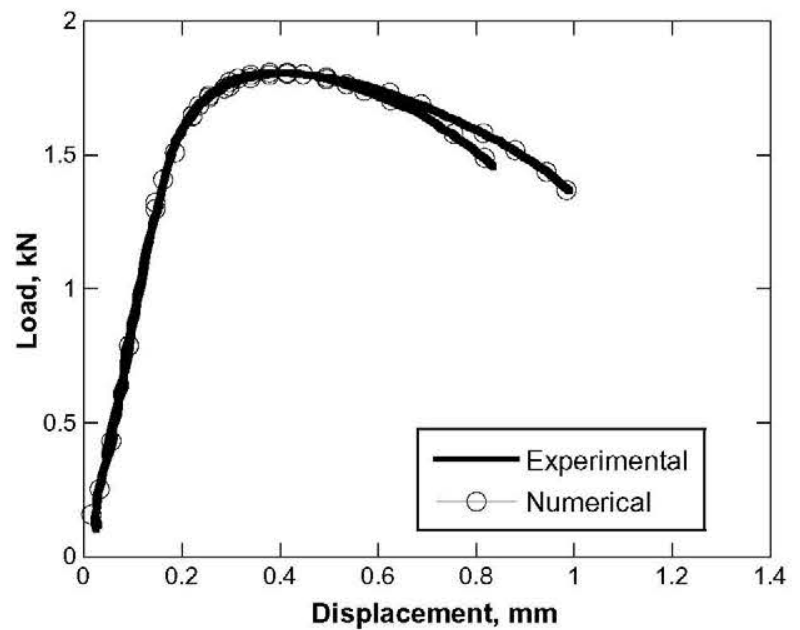

Fig. 15. Experimental and numerical load vs. displacement curve, $250 \mathrm{wppm}$. 


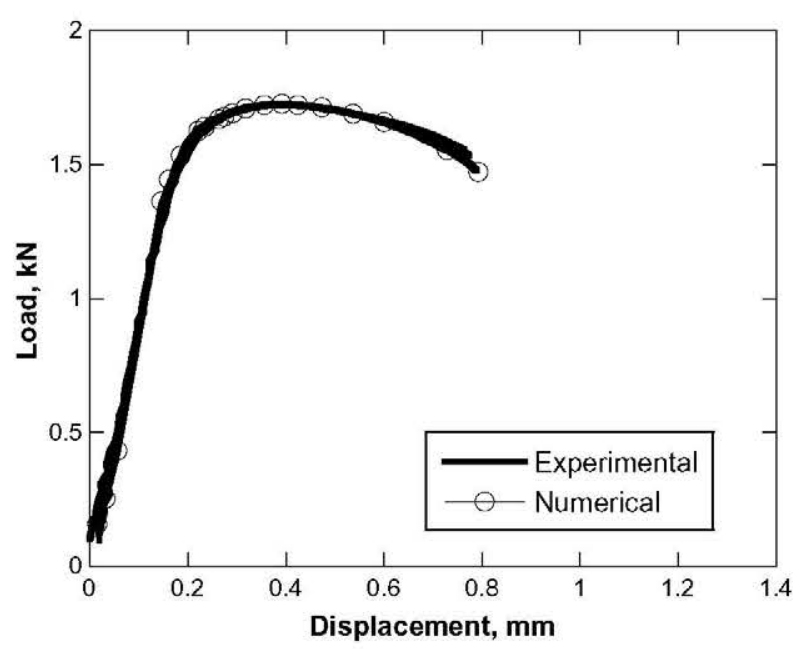

Fig. 16. Experimental and numerical load vs. displacement curve, $500 \mathrm{wppm}$.

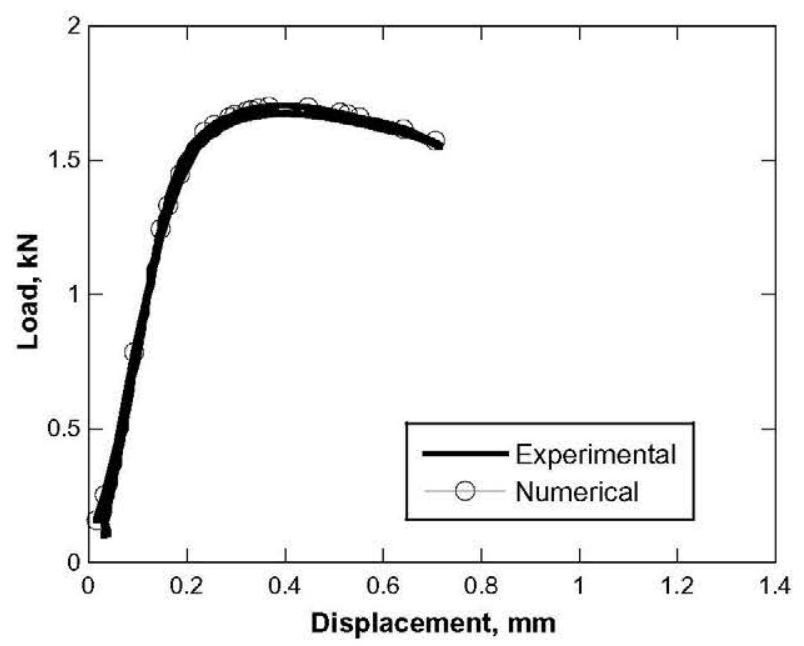

Fig. 17. Experimental and numerical load vs. displacement curve, $1200 \mathrm{wppm}$.

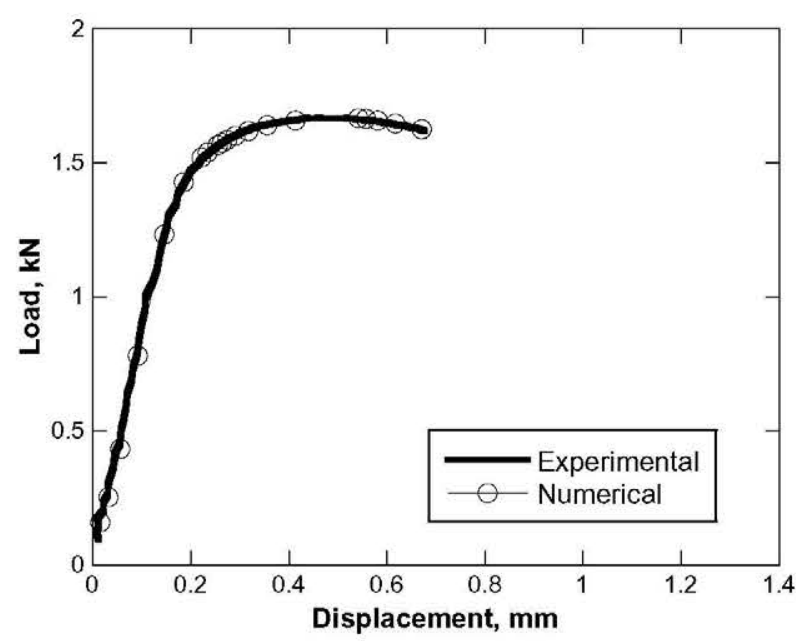

Fig. 18. Experimental and numerical load vs. displacement curve, $2000 \mathrm{wppm}$.

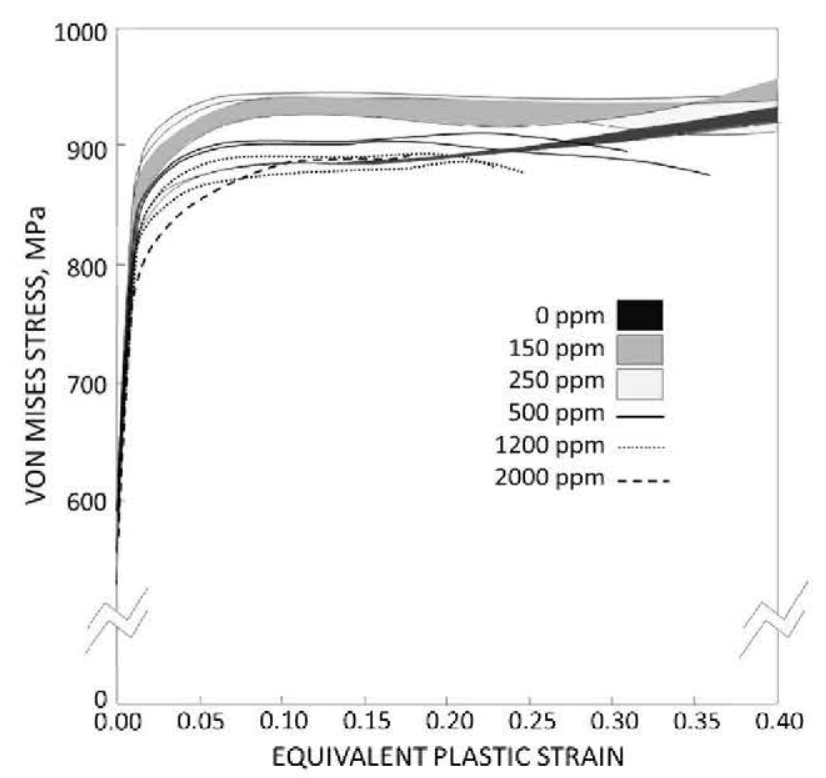

Fig. 19. Calculated Von Mises stress vs. equivalent plastic strain, $0-2000 \mathrm{wppm}$.

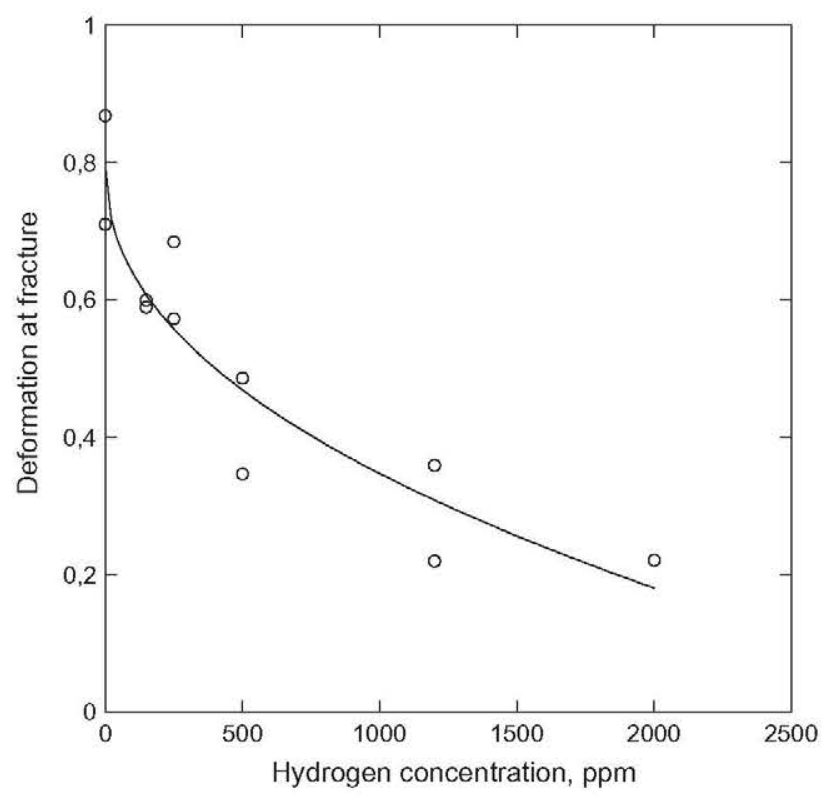

Fig. 20. Deformation at fracture as function of the hydrogen concentration.

similar. Thus, it seems that the mechanical properties of the cladding samples do not depend strongly on the hydrogen concentration at $20^{\circ} \mathrm{C}$. In addition, no noticeable strain hardening occurs.

The deformation at fracture and the area reduction (Eqs. (3) and (4)) are represented in Figs. 20 and 21 as a function of the hydrogen content. Scattering is significant in both cases. The ductility decreases with the hydrogen concentration, this being clearly noticeable from $500 \mathrm{wppm}$ hydrogen. The deformation at fracture for a sample with $2000 \mathrm{wppm}$ is $70 \%$ lower than the value for an as-received sample. Such results are consistent with the behaviour observed in the load vs. displacement records and the fractographic observations. As was explained above, the fracture micromechanisms are quite different for samples without (as-received) and with $2000 \mathrm{wppm}$ of hydrogen. In the former case nucleation, growth and coalescence of microavoids is observed, which is typical of ductile fracture. On the contrary, quasi-cleavage is found for sam- 


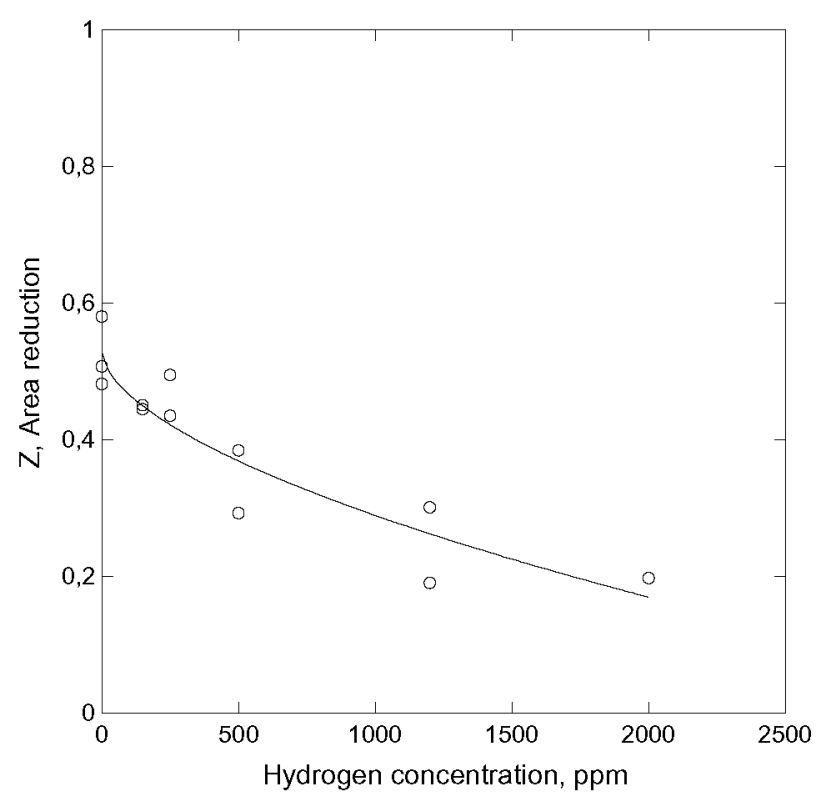

Fig. 21. Area reduction as function of the hydrogen concentration.

ples with 2000 wppm hydrogen, which is associated to brittle fracture.

\section{Conclusions}

Ring tensile test were performed on unirradiated hydrogencharged samples with different hydrogen concentrations (from 0 to $2000 \mathrm{wppm}$ ). It was found that this test was insensitive to low hydrogen concentrations. In fact, the maximum load does not change significantly over the range of hydrogen concentrations investigated. Only a slight decrease is found for $2000 \mathrm{wppm}$. The measured displacement at fracture shows a noticeable decrease from $500 \mathrm{wppm}$ to $2000 \mathrm{wppm}$, which suggests a lower ductility of the material in the presence of hydrides. In fact, this is confirmed by the deformation at fracture and the area reduction, with the decrease in the ductility being clearly noticeable from $500 \mathrm{wppm}$ hydrogen.

Observation of the fracture surfaces shows a ductile fracture micromechanism for as-received samples (nucleation, growth and coalescence of microvoids), a brittle one (quasi-cleavege) for samples with 2000 wppm hydrogen, which is consistent with the behaviour observed in the mechanical tests.

A new method was proposed to obtain the mechanical properties of the cladding in the hoop direction from the ring tensile tests. In this case, non-linear geometry is considered, and an iterative procedure is used so universal curves are not needed. The agreement with the experimental results after only five iterations is excellent for all hydrogen concentrations investigated.

The calculated stress-strain curves show that the mechanical properties of the unirradiated cladding in the hoop direction do not depend on the hydrogen concentration (from 0 to $2000 \mathrm{wppm}$ ). It was found that the material does not show a noticeable strain hardening in the hoop direction, regardless of the hydrogen content

\section{Acknowledgments}

The authors wish to acknowledge the financial support received from the Ministerio de Educación y Ciencia (Project ENE2005-06478/ CON), and ENUSA, ENRESA and CSN for collaboration and support in the project. This work was carried out within the framework of the projects DUMEINPA, supported by the Comunidad de Madrid, and SEDUREC, from the national research project CONSOLIDERINGENIO 2010.

\section{References}

[1] S. Arsene, J.B. Bai, Effet de la microstructure et de la temperature sur la transition ductile-fragile des zircaloy hydrures, PhD disertation, École Centrale Paris, 1997.

[2] M. Grange, Fragilisasion du zircaloy par l'hydrogene: comportement, mécanismes d'endommagement, interaction a vec la couche d'oxide, simulation numerique, Ph.D. dissertation, École de Mines de Paris, 1998.

[3] G. Bertolino, G. Meyer, J. Perez-Ipiña, Journal of Nuclear Materials 320 (2003) 272-279.

[4] M.A. Martín-Rengel, Integridad estructural de vainas de combustible nuclear en condiciones de almacenamiento temporal en seco, ENRESA technical publication 05-2010 (in Spanish).

[5] J. Desquines, B. Cazalis, Ch. Poussard, X. Averty, P. Yvon, Journal of ASTM international 2 (6) (2005) (paper ID JA112465).

[6] S. Arsene, J.B. Bai, Journal of Testing and Evaluation 26 (1998) 26-30.

[7] S. Arsene, J.B. Bai, Journal of Testing an Evaluation 24 (1996) 386-391.

[8] S. Chang-Sun, Bong-kook, K. Linga, Engineering Failure Analysis 13 (2006) 389397.

[9] George P. Sabol, Journal of ASTM international 2 (2) (2005) (paper ID JAI12942). 